\title{
A mathematical model of healthy and dystrophic skeletal muscle biomechanics
}

\author{
Marco Stefanatia,*, Chiara Villa ${ }^{b}$, Yvan Torrente ${ }^{b}$, José Félix Rodriguez Matas ${ }^{a}$ \\ a Department of Chemistry, Materials and Chemical Engineering “Giulio Natta”, Politecnico di Milano, Piazza Leonardo da Vinci, Milan \\ 20133, Italy \\ b Stem Cell Laboratory, Department of Pathophysiology and Transplantation, Università degli Studi di Milano, Fondazione IRCCS Ca' \\ Granda Ospedale Maggiore Policlinico, Milan, Italy
}

\section{A R T I C L E I N F O}

\section{Article history:}

Received 14 June 2019

Revised 19 September 2019

Accepted 30 September 2019

Available online 1 October 2019

\section{Keywords:}

Skeletal muscle model

Skeletal fiber model

Skeletal mathematical model

Muscle mathematical model

Diaphragm mathematical model

C57Bl And mdx diaphragm mathematical model

Mouse diaphragm mathematical model

\begin{abstract}
A B S T R A C T
Duchenne Muscular Dystrophy (DMD) is a common X-linked disease, caused by mutations in the gene encoding dystrophin and characterized by widespread muscle damage that invariably leads to paralysis and death. Lack of dystrophin in the muscles of DMD patients determines an increased fragility of muscle fibers, leading to repeated cycles of necrosis and regeneration that result in failed regeneration, increased fibrosis and progressive loss of muscle function.

In this work, we propose a three-dimensional chemo-mechanical mathematical model of skeletal muscle in DMD. This model is based on stress-strain mechanical data of the muscle and studies of changes in fiber structure and interaction aiming to shade light into the biophysical mechanisms regulating muscle contraction. The results show that the model is able to reproduce the experimental data of maximum isometric force, maximum contraction velocity and concentric normalized F-V curve for the healthy and dystrophic muscle. Furthermore, the model is capable of predicting the force-velocity response of the muscle to eccentric loading without explicitly imposing its functional form in the formulation, and it is able to reproduce the concentric normalized F-V curve of the healthy fiber, as an additional proof of the predictive capabilities of the model. The resulting model represents a novel approach to study DMD pathogenesis by providing insights into the underlying mechanisms of muscle response to force associated with the impaired muscle functionality. Moreover, it could be an innovative tool for researchers to predict muscle response under conditions that are not possible to explore in the laboratory and an important step towards a new paradigm of in-silico trials that could allow identifying novel therapies bypassing the use of animal models.
\end{abstract}

(c) 2019 Elsevier Ltd. All rights reserved.

\section{Introduction}

DMD is a devastating muscle degenerative disease caused by a mutation in the dystrophin gene (Hoffman et al., 1987). Dystrophin is a cytoskeletal protein essential for the stability of multinucleated myofiber membrane in skeletal muscle (Durbeej and Campbell, 2002), whose lack results in increased fragility of the sarcolemma (Petrof et al., 1993a). DMD patients suffer from progressive loss of muscle function to paralysis and death in the third decade of life (Emery, 2002). The

\footnotetext{
* Corresponding author.

E-mail address: marco.stefanati@polimi.it (M. Stefanati).
} 
chronic nature of the disease with continuous cycles of muscle degeneration/regeneration determines unsuccessful muscle regeneration, typically characterized by persistent myofiber degeneration, inflammation and fibrosis, which is essentially an excessive accumulation of extracellular matrix (ECM) components contributing to muscle weakness.

The mdx mice (Kumamoto et al., 2000) is one of the mostly used animal models of DMD. However, most skeletal muscles of the mdx mouse show little fibrosis and functional alterations, which is in striking contrast to the destructive muscle disease found in humans with the same genetic defect. A notable exception to this mild muscle phenotype observed in mdx mice is the diaphragm (DIA) (Krüger and Kloetzel, 2012; Stedman and Sweeney, 1991; Tidball and Wehling-Henricks, 2007), which exhibits significant fibrosis as well as greatly impaired contractile function from an early age, and is regarded as a close phenotype to the human dystrophic muscle. The diaphragm muscle of mdx mice undergoes a progressive necrosis and fibrosis throughout the life of the animal similar to that occurring in muscles of humans with DMD, leading to severe muscle atrophy, weakness and abnormal mechanical properties (Attal et al., 2000).

Bates et al. (2013) found that stress was smaller for mdx than control at the muscle strip level but was not different at the single fiber level. This weakness was only observed at the level of the intact muscle bundle and could not be narrowed down to a specific mechanical impairment of its individual fibers or myosin molecules. Moreover, Bates et al. reported little differences of contraction force between healthy and dystrophic fibers ( $21.1 \pm 7.5 \mathrm{kPa}$ for $\mathrm{mdx}, 25.9 \pm 9.8 \mathrm{kPa}$ for wild type). Results from Bates et al. are in agreement with observations made by Friedrich et al. (2010) in which they used second harmonic generation imaging to reveal vastly altered subcellular sarcomere microarchitecture in intact single dystrophic mdx muscle cells ( 1 year old). Their results showed that misorientations (tilting, twisting and local axis deviations) from adjacent myofibrils could account for force detriments in mdx fibers during unsynchronized contractile activation. Therefore, the dystrophic process results in remodeling of the muscle microarchitecture without affecting molecular motorprotein interactions. Similarly, the concentric isotonic force-velocity contraction for dystrophic muscle is lower than for healthy muscle. In particular, reduction of up to 38\% has been reported for DIA (Coirault et al., 1999; 2003), of 22\% for SOL (Lynch et al., 2000), of $15 \%$ for SH (Attal et al., 2000) and 1.3\% for EDL (Lynch et al., 2000). This reduction in shortening velocity and contraction force in the dystrophic diaphragm could be connected to the reduction in the percentage of faster type fiber isotypes and the increase in amount of slower type fiber isotypes (Muller et al., 2001), added to the increment in the amount of ECM. This is supported by the observation that, in mdx DIA, the difference in unitary force produced per cross-bridge between control and dystrophic mdx mice is about $5 \%$, whereas fat and connective tissue infiltration represent about $20 \%$ of the total muscle area (Barbin et al., 2016; Farini et al., 2007).

Hence, it can be argued that the dystrophic process does not affect isolated actin-myosin (cross-bridge kinetics) interactions and the reduced force in mdx muscle probably involves other mechanisms, i.e., excitation contraction coupling and morphological microstructure alterations (the latter at least for the age group and muscle type examined in their study) (Friedrich et al., 2010).

Significant advances in the mathematical model of skeletal muscle have been produced in the last two decades (Böl and Reese, 2008; Grasa et al., 2011; Heidlauf and Röhrle, 2014; Hernández-Gascón et al., 2013; Virgilio et al., 2015). These models simulate the dynamic behavior of muscles with mathematical equations inspired by muscle contractile processes (cross-bridge mechanisms), coupling chemical reaction equations governing cross-bridge kinetics with cross-bridge distortion responsible for the active force generation. However, most mathematical models of active muscle behavior have been developed for healthy muscle, with little efforts regarding the application to DMD skeletal muscle. Current modeling on dystrophic muscle is limited to study the relationship between microstructural disease adaptations and modifications in the mechanical properties of muscle (passive) and strain in the cell membrane, not accounting for chemo-mechanical interactions and ignoring the active response of the muscle (Petrof et al., 1993b).

The aim of this work is to develop a comprehensive chemo-mechanical model of muscle-skeletal tissue amenable for modeling dystrophic skeletal muscle. The model accounts for the contribution of individual fibers and the extracellular matrix. The main supported hypothesis behind is that, the myosin function, as well as the single fiber mechanics, do not underlie the weakness of the dystrophic muscle (Bates et al., 2013; Friedrich et al., 2010) and, consequently, the muscle active part (cross-bridge mechanisms inside myofibril) is not seriously affected by the dystrophic disease. So, it is hypothesized that the loss of isometric contraction force and isotonic concentric contraction velocity is a mechanical problem resulting from increase of muscle matrix stiffness, loss of number and density of fibers, decrease of lateral transmission force efficiency (Ramaswamy et al., 2011), non-uniform fiber distribution (Blemker et al., 2005) and myofibrillar structure misalignment (Schneidereit et al., 2018). In this work, we are going to test this hypothesis and verify the Bates et al. (2013) hypothesis that the force on non-necrotic fibers in dystrophic muscle is not affected by dystrophy. Even though the proposed model is generally applicable to muscle-skeletal tissue, we have specified the model to healthy and mdx DIA in an effort to understand the potentialities of the proposed model to study DMD disease. The choice of the mdx DIA results from the fact that the phenotype of this muscle results closer to the human phenotype.

\section{Methods}

\subsection{Experimental settings}

Uniaxial tests have been used to determine the passive stress-strain response of the diaphragm of C57Bl (wild-type) and mdx mice. This experiments will be used to characterize the mechanical behavior of the extracellular matrix within the 


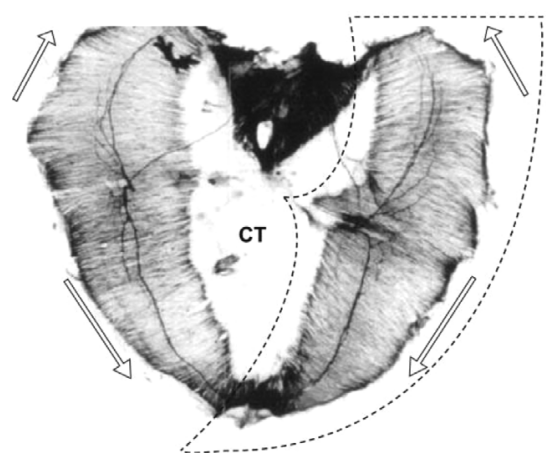

(a)

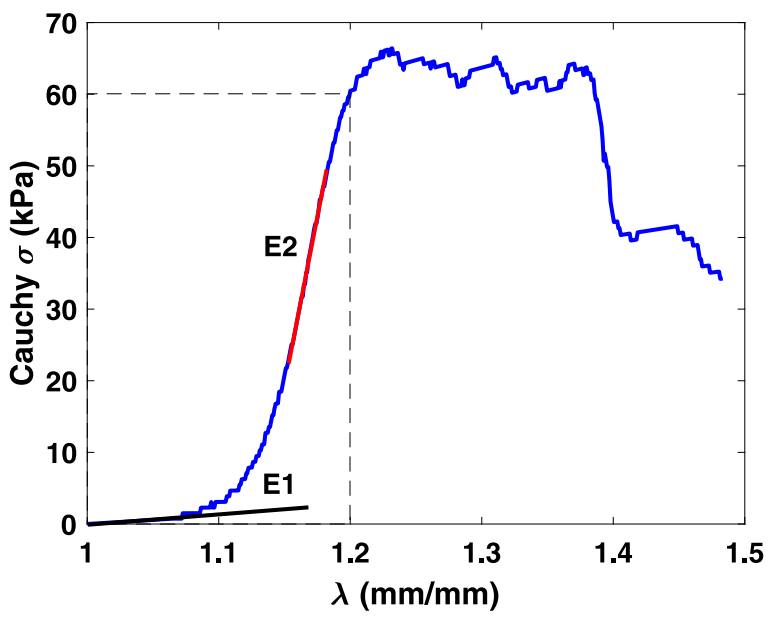

(b)

Fig. 1. (a) DIA muscle structure: the arrow indicates the stretching direction during the uniaxial test. (b) Typical stress-strain curve for C57Bl mice. Initial and large strain tangent modulus were characterized as $E_{1}$ and $E_{2}$. Tangent modulus at large strain was measured at $15 \%$.

mathematical model. It is important to remark that, even though a significant amount of work has been performed to asses the active response of the diaphragm, a limited number of studies comprise the passive behavior and non of these reports the full stress-strain behavior (Hakim et al., 2011; Smith and Barton, 2014).

A total of $N=5 \mathrm{mdx}$ mice and $N=5$ C57Bl mice, age 14 weeks, were supplied by Charles River Laboratories (Calco, Lecco, Italy). All procedures used for the extraction and harvesting of samples were approved by the Ethics Committee of Fondazione IRCCS Ca' Granda Ospedale Maggiore Policlinico, Milan. According to the protocol, all mice were maintained in a supervised laboratory animal facility in polypropylene shoebox cages. Mice were fed a standard pelleted diet ad libitum and were allowed to continuous water access. Mice were sacrificed by carbon dioxide narcosis followed by cervical dislocation (Farini et al., 2016).

Muscle biopsies were taken from diaphragm mice and muscle strips were extracted along the transverse direction of the fibers (see the arrow in Fig. 1a) in order to analyze the ECM mechanical properties of the wild-type and mdx DIA muscle. Sample dimensions (length, width, and thickness) were determined from digital imaging of the sample once clamped to the testing machine. For this purpose, we used the imaging software ImageJ. To keep the hydration of the sample during the test, a humidifier was used. The uniaxial tensile test was conducted on a BOSE ElectroForce 3200 electromagnetic testing machine under traction displacement control at a strain rate of $0.01 \mathrm{~mm} /(\mathrm{mm} \cdot \mathrm{s})$ to preserve quasi-static conditions. Five preconditioning loading-unloading cycles up to a $20 \%$ strain (at a frequency of $0.25 \mathrm{~Hz}$ ) were conducted on each sample before applying uniaxial traction loading until rupture.

\subsection{Mathematical model}

The mathematical model of skeletal muscle is based on the sarcomere model presented by Razumova et al. (1999) and Campbell et al. (2000). The model is formed of two parts: (i) the myofilament $\left[\mathrm{Ca}^{2+}\right]$ kinetics and cross-bridge force generation (see Fig. 2a), and (ii) the mechanical model of the muscle composed of the active response of the fiber and the passive response of ECM (see Fig. 2b). In the present model, the force-length dependence and the force-velocity dependence are not explicitly defined in the expression of the active stress, as occurs in most traditional models of skeletal muscle (Blemker et al., 2005; Böl and Reese, 2008; Heidlauf and Röhrle, 2014; Hernández-Gascón et al., 2013). In this model the force-length dependence is incorporated in the kinetics of the cross-bridge force generation, where as the force-velocity response is a consequence of the dynamics defining the active response of the fiber. This structure allows an easier physical interpretation of the mechanism behind the muscle contraction dynamics.

\subsubsection{Myofilament kinetics and cross-bridge force generation}

Sarcomere force generation depends on the number of cross-bridges (XB) in a force bearing state, which depends on the sarcomere deformation, the $\left[\mathrm{Ca}^{2+}\right]$ activation, thin filament regulation and cross-bridge cycling. For the skeletal muscle, this process can be represented by a reduced three state model shown in Fig. 2a. The thin-filament regulatory unit may be in the off position $R_{\text {off }}$ or the on position, with switching between on and off being governed by the rate on, $k_{\text {on }}$, and off, $k_{\text {off }}$ coefficients. These rate coefficients depend on the intracellular $\left[\mathrm{Ca}^{2+}\right]$ (see Fig. 2a). The cross-bridge kinetics is described by the reduced three state model. A state in which the myosin heads are detached from actin filament $(D)$, a state in which the myosin heads are attached to actin filament in a weak load bearing condition (pre-power stroke $A_{1}$ ) and a state in which the 


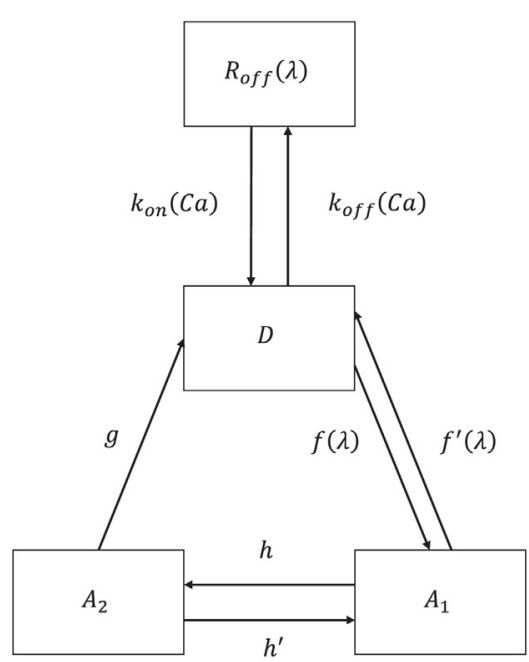

(a)

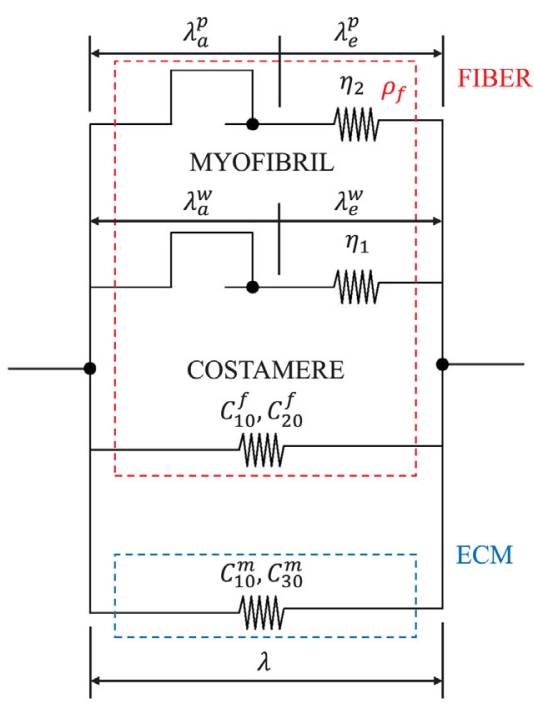

(b)

Fig. 2. Two main elements of the muscle model. (a) $\left[\mathrm{Ca}^{2+}\right]$ kinetics described by a reduced three state model. (b) Mechanical representation of the muscle. The mechanical model is composed of a contractile element describing the cross-bridge force generation and a passive element describing the elastic response of the ECM.

myosin heads are attached to actin filament in a power load bearing condition (post-power stroke $A_{2}$ ). Attachment, power stroke and detachment occur cyclically. This model is described by a set of three non-linear ordinary differential equations (Razumova et al., 1999).

$$
\begin{aligned}
& \frac{d D(t)}{d t}=k_{o n} \cdot R_{o f f}(t)+f^{\prime} \cdot A_{1}(t)+g \cdot A_{2}(t)-\left[k_{o f f}+f\right] \cdot D(t), \\
& \frac{d A_{1}(t)}{d t}=f \cdot D(t)+h^{\prime} \cdot A_{2}(t)-\left[f^{\prime}+h\right] \cdot A_{1}(t), \\
& \frac{d A_{2}(t)}{d t}=h \cdot A_{1}(t)-\left[h^{\prime}+g\right] \cdot A_{2}(t)
\end{aligned}
$$

where $R_{o f f}(t, \lambda)=R_{o v}(\lambda)-D(t)-A_{1}(t)-A_{2}(t)$ and $R_{o v}(\lambda)$ represents the total number of cross-bridges for a given filament overlap, function of the sarcomere length. Rate constants $g, f, f^{\prime}, h$ and $h^{\prime}$ govern steps in the cycles, with primes designating reverse reactions. Isometric force is generated as cross-bridges go through the power stroke (states $A_{1}$ and $A_{2}$ ). The definition and equations of the different rate constants are given in the Appendix A.

Similarly to Campbell et al. (2001), the cross-bridge force generation is derived from a deformation balance that accounts for distortion due to cross-bridge cycling, and for distortion imposed by shear motion between thick and thin filaments in both the weak and power stroke states $A_{1}$ and $A_{2}$ respectively. This translates mathematically into two non-linear first order differential equations governing the active stretches $\lambda_{a}^{w}$ (pre-power stroke) and $\lambda_{a}^{p}$ (post-power stroke) associated with the fiber (see Fig. 2b). Let $s_{1}(t)=\ln \left(\lambda_{a}^{w}\right)$ and $s_{2}(t)=\ln \left(\lambda_{a}^{p}\right)$,

$$
\begin{aligned}
& \frac{d s_{1}(t)}{d t}=\left(f \cdot \frac{D(t)}{A_{1}(t)}+h^{\prime} \cdot \frac{A_{2}(t)}{A_{1}(t)}\right) \cdot\left[\ln (\lambda)-s_{1}(t)-s_{r}^{w}\right], \\
& \frac{d s_{2}(t)}{d t}=h \cdot \frac{A_{1}(t)}{A_{2}(t)} \cdot\left[\ln (\lambda)-s_{2}(t)-s_{r}^{p}\right],
\end{aligned}
$$

where $f, h$ and $h^{\prime}$ are rate constants shown in Table 2, and $s_{r}^{w}=0.0006, s_{r}^{p}=0.0373$ are the cross-bridge deformation in steady state conditions. The Eq. (4) were modified from those reported in Razumova et al. (1999) in the sense that distortions are defined as logarithmic strains in order to account for finite deformations, following an approach similar to Khayyeri et al. (2016). Note, that using the logarithmic strain in the formulation is equivalent to expressing the total stretch $\lambda$ as a multiplicative split $\lambda=\lambda_{a} \cdot \lambda_{e}$, with $x(t)=\ln \left(\lambda_{e}\right)$ (Hernández-Gascón et al., 2013; Stålhand et al., 2011). Further, in our model, as in Razumova et al., the half-sarcomere average distortion, $\ln (\lambda)$, is composed of an active component $s(t)=\ln \left(\lambda_{a}\right)$ and an elastic component $x(t)=\ln (\lambda)-s(t)$. However, while Razumova et al. reported the equations of crossbridge distortion as a function of $x(t)$, where $d x(t) / d t=d S L(t) / d t-d s(t) / d t$ with SL the half-sarcomere length, we have chosen to express Eq. (4) as a function of the active component $s(t)$ instead. 


\subsubsection{Mechanical model}

The mechanical model of the muscle is shown in Fig. 2b. The model consists of an active element (fiber) in parallel with a passive elastic element associated with the ECM. The fiber is composed of two elements: (i) the myofibril consisting on an active contractile element (myotubes) in series with an elastic element, and (ii) the costamere modeled as another elastic element in parallel with myofibril. The fiber active response is related to actin-myosin sliding interaction, whereas the fiber passive response is principally due to the elastic response of the cross-bridges and titin (Hernández-Gascón et al., 2013).

A frequently used methodology to formulate an elastic constitutive law for fibred soft tissues consists in postulating the existence of a strain energy function (SEF) (Simo and Taylor, 1991) from which the stresses can be derived. For soft tissues, the SEF is decoupled into a volumetric and volume preserving parts in order to handle the quasi-incompressible constraint. In addition, in the case of the skeletal muscle the SEF is also a function of the active stretches $\lambda_{a}^{w}$ and $\lambda_{a}^{p}$, and the structural tensor $\mathbf{N}=\mathbf{n}_{0} \otimes \mathbf{n}_{0}$ which defines the anisotropy of the tissue do to the fibers,

$$
W\left(\mathbf{C}, \mathbf{N}, \lambda_{a}^{w}, \lambda_{a}^{p}\right)=U(J)+\bar{W}\left(\overline{\mathbf{C}}, \mathbf{N}, \lambda_{a}^{w}, \lambda_{a}^{p}\right),
$$

where $\mathbf{C}=\mathbf{F}^{T} \mathbf{F}$ is the right Cauchy-Green deformation tensor, with $\mathbf{F}$ the deformation gradient, $J=\operatorname{det} \mathbf{F}$ is the Jacobian and $\overline{\mathbf{C}}=J^{-2 / 3} \mathbf{C}$ is the modified right Cauchy-Green deformation tensor.

For the skeletal muscle, the deviatoric part of the strain energy function is decoupled into a strain energy function related to the matrix and a strain energy function related to fiber as,

$$
\bar{W}\left(\overline{\mathbf{C}}, \mathbf{N}, \lambda_{a}^{w}, \lambda_{a}^{p}\right)=\bar{W}_{\text {matrix }}(\overline{\mathbf{C}})+\rho_{f} \cdot \bar{W}_{f}\left(\overline{\mathbf{C}}, \mathbf{N}, \lambda_{a}^{w}, \lambda_{a}^{p}\right),
$$

where $\rho_{f}$ is the cross section area fiber density (CSA fiber density) defined as the ratio between the total fiber area $A_{f}$ and muscle area $A_{\text {musc }}\left(\rho_{f} \leq 1\right)$. Further, the strain energy function of the fiber is divided into a passive contribution, due to the costamere, and an active contribution associated with the muscular actin-myosin system,

$$
\bar{W}_{f}\left(\overline{\mathbf{C}}, \mathbf{N}, \lambda_{a}^{w}, \lambda_{a}^{p}\right)=\bar{W}_{f, p}(\overline{\mathbf{C}}, \mathbf{N})+\bar{W}_{f, a}\left(\overline{\mathbf{C}}, \mathbf{N}, \lambda_{a}^{w}, \lambda_{a}^{p}\right) .
$$

In order to guarantee frame invariance, the SEF is expressed in terms of the irreducible integrity bases of the tensors $\overline{\mathbf{C}}$ and $\mathbf{N}$,

$$
\bar{I}_{1}=\operatorname{tr} \overline{\mathbf{C}}, \quad \bar{I}_{2}=\frac{1}{2}\left[(\operatorname{tr} \overline{\mathbf{C}})^{2}-\operatorname{tr} \overline{\mathbf{C}}^{2}\right], \quad \bar{I}_{3}=\operatorname{det} \overline{\mathbf{C}}=1, \quad \bar{I}_{4}=\overline{\mathbf{C}}: \mathbf{N}=\lambda^{2} .
$$

Hence,

$$
\begin{aligned}
& \bar{W}_{\text {matrix }}(\overline{\mathbf{C}})=\bar{W}_{\text {matrix }}\left(\bar{I}_{1}, \bar{I}_{2}\right), \\
& \bar{W}_{f, p}(\overline{\mathbf{C}}, \mathbf{N})=\bar{W}_{f, p}\left(\bar{I}_{1}, \bar{I}_{2}, \bar{I}_{4}\right), \\
& \bar{W}_{f, a}\left(\overline{\mathbf{C}}, \mathbf{N}, \lambda_{a}^{w}, \lambda_{a}^{p}\right)=\bar{W}_{f, a}\left(\bar{I}_{1}, \bar{I}_{2}, \bar{I}_{4}, \lambda_{a}^{w}, \lambda_{a}^{p}\right) .
\end{aligned}
$$

With the strain energy function so defined, it is possible to derive the second Piola-Kirchhoff stress tensor as,

$$
\mathbf{S}=2 \frac{\partial W}{\partial \mathbf{C}}
$$

with the Cauchy stress tensor obtained as the weighted push-forward i.e., $\sigma=J^{-1} \mathbf{F S F}^{T}$.

The passive behavior associated with the matrix, $\bar{W}_{\text {matrix }}$, and the fibers, $\bar{W}_{f, p}$, can be modeled using any strain energy function available for soft tissues. In particular, we have assumed the matrix to behave isotropically, whereas the passive behavior of the fibers is assumed to be anisotropic (Hernández-Gascón et al., 2013; Stålhand et al., 2011). For the active behavior, $\bar{W}_{f, a}$ is assumed as the sum of the contribution of the two states of attached XBs: $A_{1}$ (attached pre-power stroke state) and $A_{2}$ (attached post-power stroke state) (Campbell et al., 2001),

$$
\begin{aligned}
\bar{W}_{f, a}\left(\bar{I}_{4}, \lambda_{a}^{w}, \lambda_{a}^{p}\right)= & \frac{1}{2} \eta_{1} A_{1}\left[\frac{1}{2} \ln \left(\bar{I}_{4}\right)-\ln \left(\lambda_{a}^{w}\right)\right]^{2} \\
& +\frac{1}{2} \eta_{2} A_{2}\left[\frac{1}{2} \ln \left(\bar{I}_{4}\right)-\ln \left(\lambda_{a}^{p}\right)\right]^{2},
\end{aligned}
$$

where $\eta_{1}$ and $\eta_{2}$ are elastic constants for the pre- and post- power stroke states. The two active elements in parallel (see Fig. 2b), indicate that inside the muscle some fiber/sarcomere may be in pre-power stroke state $\left(A_{1}\right)$ and other in postpower stroke state $\left(A_{2}\right)$, depending on the probabilities $A_{1}$ and $A_{2} . A_{1}$ is the likelihood that myosin heads are in pre-power stroke state, while $A_{2}$ is the likelihood that myosin heads are in post-power stroke state. 
Table 1

Data used for parameter fitting and for predictive capacity verification.

\begin{tabular}{ll}
\hline Fitting data & Reference \\
\hline Fiber passive response & (Granzier and Irving, 1995) \\
Matrix passive response & Performed experimental test (Section 3.2) \\
CSA fiber density & (Barbin et al., 2016; Farini et al., 2007) \\
Muscle C57Bl and mdx Concentric F-V curve & (Coirault et al., 2003) \\
Muscle C57Bl and mdx Isometric stress & (Bates et al., 2013) \\
Muscle C57Bl and mdx Concentric velocity & (Coirault et al., 1999; 2003) \\
Verification data & Reference \\
\hline Fiber C57Bl Concentric F-V curve & (Bottinelli et al., 1991; Edman, 2005) \\
Muscle C57Bl Eccentric F-V curve & (Till et al., 2008) \\
Fiber C57Bl Isometric stress & (Pellegrino et al., 2003) \\
Fiber C57Bl Concentric velocity & (Pellegrino et al., 2003) \\
\hline
\end{tabular}

\subsubsection{One-dimension particularization}

Let us assume that muscle contraction occurs in one-dimension. This formulation results particularly useful when performing model parameter identification. The strain energy can now be expressed as,

$$
\begin{aligned}
W^{1 D}\left(\lambda, \lambda_{a}^{w}, \lambda_{a}^{p}\right) & =W_{\text {matrix }}^{1 D}(\lambda)+\rho_{f} W_{f}^{1 D}\left(\lambda, \lambda_{a}^{w}, \lambda_{a}^{p}\right), \\
& =W_{\text {matrix }}^{1 D}(\lambda)+\rho_{f}\left[W_{f, p}^{1 D}(\lambda)+W_{f, a}^{1 D}\left(\lambda, \lambda_{a}^{w}, \lambda_{a}^{p}\right)\right],
\end{aligned}
$$

where $W_{\text {matrix }}^{1 D}$ and $W_{f}^{1 D}$ represent the deviatoric part associated with the extracellular matrix and fiber, respectively. The active response of the fiber reduces to,

$$
\begin{aligned}
W_{f, a}^{1 D}\left(\lambda, \lambda_{a}^{w}, \lambda_{a}^{p}\right)= & \frac{1}{2} \eta_{1} A_{1}\left[\ln \lambda-\ln \left(\lambda_{a}^{w}\right)\right]^{2} \\
& +\frac{1}{2} \eta_{2} A_{2}\left[\ln \lambda-\ln \left(\lambda_{a}^{p}\right)\right]^{2} .
\end{aligned}
$$

For the passive behavior of the fibers and ECM, the following phenomenological SEFs are assumed,

$$
\begin{aligned}
W_{\text {matrix }}^{1 D} & =C_{10}^{m}\left(\bar{I}_{1}-3\right)+C_{30}^{m}\left(\bar{I}_{1}-3\right)^{3}, \\
W_{f, p}^{1 D} & =C_{10}^{f}\left(\bar{I}_{1}-3\right)+C_{20}^{f}\left(\bar{I}_{1}-3\right)^{2}+\frac{k_{1}^{f}}{2 k_{2}^{f}}\left[e^{k_{2}^{f}\left(\bar{I}_{4}-1\right)^{2}}-1\right],
\end{aligned}
$$

where $\bar{I}_{1}=\lambda^{2}+\frac{2}{\lambda}$ and $\bar{I}_{4}=\lambda^{2}$, and $C_{10}^{m}, C_{30}^{m}, C_{10}^{f}, C_{20}^{f}, k_{1}^{f}$ and $k_{2}^{f}$ are material constants to be identified from experimental data. The SEF for the isotropic part of fibers and matrix was chosen in order to best fitting the stress-strain curves from uniaxial tensile test curves for skeletal sarcomeres reported by Granzier and Irving (1995) (see Section 3.1), and from uniaxial-test performed in our laboratory (see Sections 2.1 and 3.2).

For the model at fiber level the SEF $W^{1 D}$ is,

$$
W^{1 D}\left(\lambda, \lambda_{a}^{w}, \lambda_{a}^{p}\right)=W_{f, p}^{1 D}(\lambda)+W_{f, a}^{1 D}\left(\lambda, \lambda_{a}^{w}, \lambda_{a}^{p}\right),
$$

where $W_{\text {matrix }}^{1 D}$ is equal to 0 and $\rho_{f}$ is equal to 1 .

Instead, for the model at whole muscle level the SEF $W^{1 D}$ is,

$$
W^{1 D}\left(\lambda, \lambda_{a}^{w}, \lambda_{a}^{p}\right)=W_{\text {matrix }}^{1 D}(\lambda)+\rho_{f}\left[W_{f, p}^{1 D}(\lambda)+W_{f, a}^{1 D}\left(\lambda, \lambda_{a}^{w}, \lambda_{a}^{p}\right)\right] .
$$

The Cauchy stress tensor in the one-dimensional problem is given by,

$$
\sigma=\lambda \frac{\partial W^{1 D}}{\partial \lambda}
$$

\section{Specialization of the model}

The proposed framework can be used to model musculo-skeletal tissue in general. However, since we were interested in studying mechanisms associated with DMD, the model has been specialized for healthy and dystrophic (mdx) mice, one of the mostly used animal models of DMD (Kumamoto et al., 2000). In particular, the attention has been placed on the diaphragm, which exhibits significant fibrosis as well as greatly impaired contractile function from an early age, and is regarded as a close phenotype to the human dystrophic muscle (Stedman and Sweeney, 1991). The proposed model has been specialized using experimental data published in the literature and obtained in our laboratory. In this regard, the experimental data has been divided in two groups, one for fitting the model parameters and one for model verification, as shown in Table 1. Data related with macroscopic passive behavior, and isometric and concentric muscle contraction were 
Table 2

Parameters of the mathematical model.

\begin{tabular}{|c|c|c|c|c|c|}
\hline \multicolumn{6}{|c|}{ Parameters for cross-bridge kinetics } \\
\hline$f_{0}\left(s^{-1}\right)$ & $f_{0}^{\prime}\left(s^{-1}\right)$ & $h_{i s o}\left(s^{-1}\right)$ & $h_{i s o}^{\prime}\left(s^{-1}\right)$ & \multicolumn{2}{|l|}{$g_{0}\left(s^{-1}\right)$} \\
\hline 12.8716 & 842.3587 & 5.4744 & 3.8510 & \multicolumn{2}{|l|}{28.1755} \\
\hline$k_{o n}^{C a}\left(s^{-1}\right)$ & $k_{o f f}^{C a}\left(s^{-1}\right)$ & $k_{o n}^{0}\left(s^{-1}\right)$ & $k_{o f f}^{0}\left(s^{-1}\right)$ & \multicolumn{2}{|l|}{$f(\mathrm{Ca})$} \\
\hline 97.3595 & 43.2405 & 0 & 12800.1 & \multicolumn{2}{|l|}{0.9825} \\
\hline \multicolumn{6}{|c|}{ Structural parameters of the model } \\
\hline$L_{A}(\mu m)$ & $B(\mu m)$ & $L_{M}(\mu m)$ & \multicolumn{3}{|l|}{$L_{0}(\mu m)$} \\
\hline 1.2 & 0.2 & 1.6 & \multicolumn{3}{|l|}{1.1} \\
\hline$x_{0}(\mu m)$ & $S L_{0}(\mu m)$ & $R_{T}$ (adim.) & \multicolumn{3}{|l|}{$A_{s}\left(m m^{2}\right)$} \\
\hline 0.041 & 1.2 & $1.62 \times 10^{5}$ & \multicolumn{3}{|l|}{$3 \times 10^{-6}$} \\
\hline \multicolumn{6}{|c|}{ Mechanical parameters of fiber and muscle for healthy and dystrophic condition } \\
\hline \multicolumn{2}{|c|}{ Parameters } & fiber C57Bl & fiber MDX & Muscle C57Bl & Muscle MDX \\
\hline$\eta_{1}$ & $(\mathrm{kPa})$ & $6.2790 \times 10^{-1}$ & $5.2324 \times 10^{-1}$ & $6.2790 \times 10^{-1}$ & $5.2324 \times 10^{-1}$ \\
\hline$\eta_{2}$ & $(\mathrm{kPa})$ & $1.6358 \times 10^{1}$ & 6.8154 & $1.6358 \times 10^{1}$ & 6.8154 \\
\hline$C_{10}^{f}$ & $(\mathrm{kPa})$ & 0.5 & 0.5 & 0.5 & 0.5 \\
\hline$C_{20}^{f}$ & $(\mathrm{kPa})$ & 7.5197 & 7.5197 & 7.5197 & 7.5197 \\
\hline$k_{1}^{f}$ & $(\mathrm{kPa})$ & $2.5388 \times 10^{-1}$ & $2.5388 \times 10^{-1}$ & $2.5388 \times 10^{-1}$ & $2.5388 \times 10^{-1}$ \\
\hline$k_{2}^{f}$ & $(-)$ & $2.1335 \times 10^{-1}$ & $2.1335 \times 10^{-1}$ & $2.1335 \times 10^{-1}$ & $2.1335 \times 10^{-1}$ \\
\hline$C_{10}^{m}$ & $(\mathrm{kPa})$ & - & - & 1.5505 & $9.0172 \times 10^{-1}$ \\
\hline$C_{30}^{m}$ & $(\mathrm{kPa})$ & - & - & $1.0649 \times 10^{3}$ & $2.2674 \times 10^{3}$ \\
\hline$\rho_{f}$ & $(-)$ & - & - & 1.0 & 0.8 \\
\hline
\end{tabular}

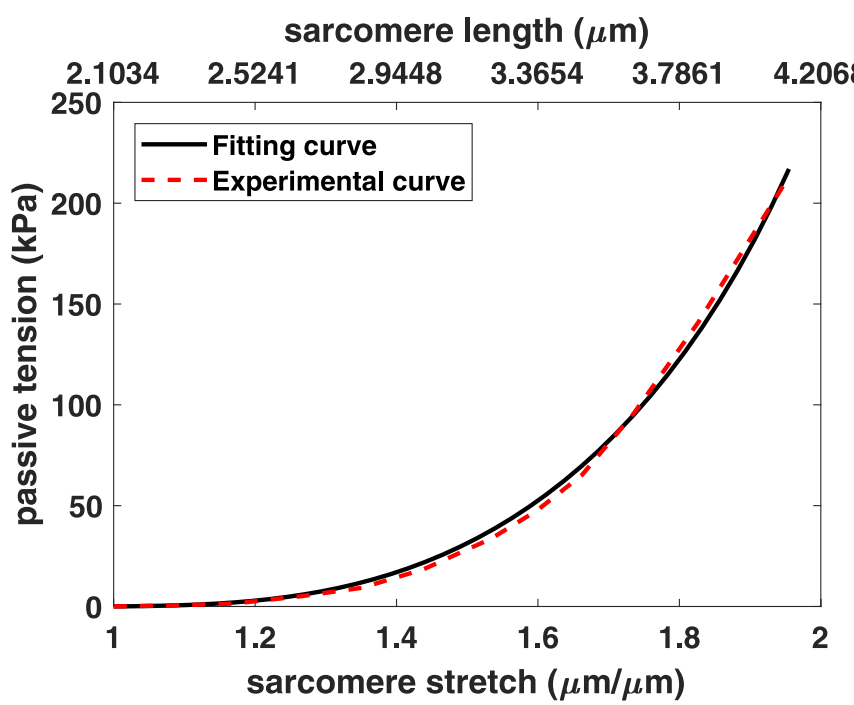

Fig. 3. Passive tension-sarcomere length relation for the skeletal muscle (Granzier and Irving, 1995).

used to identify model parameters, whereas experimental data associated with isometric and concentric fiber contraction, and with eccentric muscle contraction were used for model verification.

Model parameters can be divided in three groups: (i) cross-bridge kinetics parameters that regulate the myofilament kinetics and cross-bridge force generation, (ii) structural parameter used for the definition of the filament overlap (seeAppendix A)), and (iii) mechanical parameters associated with the elasticity of passive and active elements. The structure parameters, except $x_{0}$, were taken from Campbell et al. (2000). These parameters, shown in Table 2, were considered the same for both healthy and dystrophic fiber/muscle. The remaining cross-bridge kinetics and mechanical parameters (see Table 2), and $x_{0}$ were found by means of a nonlinear least square fitting of experimental data detailed in the following section.

\subsection{Fiber passive response}

The passive fiber model parameters $C_{10}^{f}, C_{20}^{f}, k_{1}^{f}$ and $k_{2}^{f}$ were obtained from fitting uniaxial tensile test curves for skeletal sarcomeres reported by Granzier and Irving (1995) and were assumed equal for healthy and dystrophic muscle in 


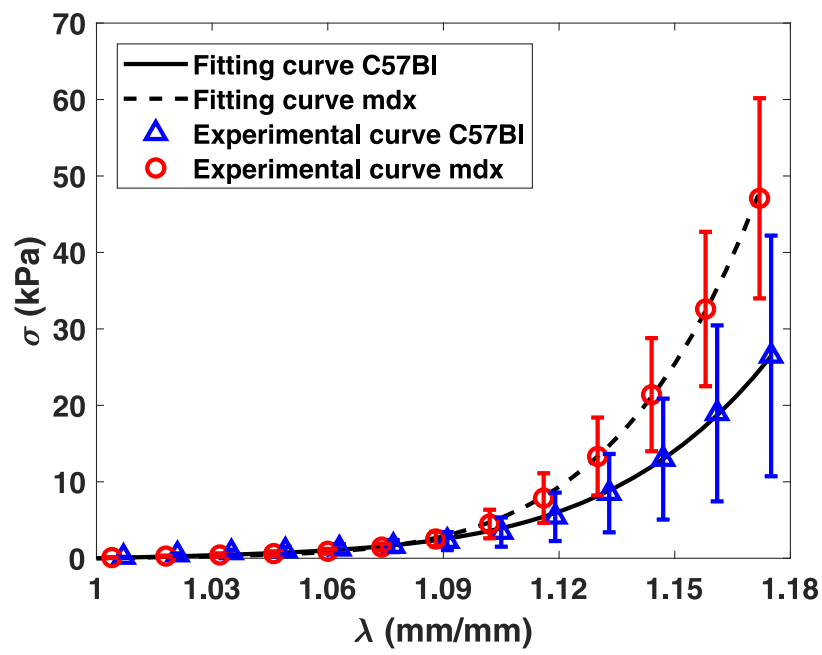

Fig. 4. Experimental passive mean stress-stretch curve of $\mathrm{C} 57 \mathrm{Bl}$ (blue triangles) and mdx diaphragm (red circles) with standard deviation and fitting model curves. (For interpretation of the references to colour in this figure legend, the reader is referred to the web version of this article.)

Table 3

Simulations results of maximum contraction stress $\left(\sigma_{\max }\right)$ in isometric contraction for single fiber and DIA muscle.

\begin{tabular}{llll}
\hline & $\begin{array}{l}\sigma_{\max } \\
(\mathrm{kPa})\end{array}$ & $\begin{array}{l}\sigma_{\max }(\%) \\
\mathrm{mdx} \text { vs C57Bl }\end{array}$ & $\begin{array}{l}\text { Experiments (kPa) } \\
\text { mean } \pm \text { SD }\end{array}$ \\
\hline fiber C57Bl & 67.70 & -58.18 & $68.7 \pm 4.3$ (Pellegrino et al., 2003) \\
fiber mdx & 28.31 & & - \\
Muscle C57Bl & 67.70 & -66.54 & $67.69 \pm 18.69$ (Bates et al., 2013) \\
Muscle mdx & 22.65 & & $22.56 \pm 1.70$ (Bates et al., 2013) \\
\hline
\end{tabular}

accordance to what reported in Bates et al. (2013) that single fiber mechanics are not affected by dystrophy. Fig. 3 shows the experimental and the model fitted curve, with parameters $C_{10}^{f}, C_{20}^{f}, k_{1}^{f}$ and $k_{2}^{f}$ reported in Table 2.

\subsection{Matrix passive response}

The material parameters for the extracellular matrix, $C_{10}^{m}$ and $C_{30}^{m}$, for healthy and dystrophic muscle were obtained by fitting average uniaxial stress-strain curves for healthy and mdx DIA performed in our laboratory. Fig. 4 shows the experimental and modeled stress-strain curves. Parameters $C_{10}^{m}$ and $C_{30}^{m}$ are reported in Table 2 (see Mechanical parameters of fiber and muscle for healthy and dystrophic condition).

\subsection{Active contraction and cross-bridges kinetics}

The CSA fiber density $\left(\rho_{f}\right)$ was determined from data of percent fibrotic area reported in literature for healthy and dystrophic DIA. Dystrophic DIA is characterized by an increase of about 20\% in fibrosis respect to the healthy DIA (Barbin et al., 2016; Farini et al., 2007). Hence, we have assumed a value of one for the healthy case (no fibrotic and fat infiltration in the muscle), and of 0.8 for dystrophic DIA. The remaining model parameters $\eta_{1}, \eta_{2}, x_{0}$ and the cross-bridges kinetics parameters $\left(f_{0}, f_{0}^{\prime}, h_{i s o}, h_{i s o}^{\prime}, g_{0}, k_{o n}^{C a}\right.$ and $k_{o f f}^{C a}$ shown in Table 2) were found by means of a constrained nonlinear fitting of normalized specific force-velocity curve for healthy and mdx mouse DIA (Coirault et al., 2003). The initial values of $x_{0}$ and the cross-bridges kinetics parameters for the nonlinear fitting were taken from Campbell et al. (2001) and correspond to mouse papillary strip. Isometric stress (Bates et al., 2013) and concentric shortening velocity of muscle (Coirault et al., 1999; 2003) were constrained to be within the range reported in the literature (see Tables 3, 4 and Fig. 5). For the dystrophic muscle, $x_{0}$ and the cross-bridges kinetics parameter were assumed equal to those of healthy muscle, since experimental observations support that the myosin function do not underlie the weakness of the dystrophic muscle (Bates et al., 2013; Friedrich et al., 2010). The constrained optimization problem was solved in MATLAB® version R2017b.

Fig. 5 shows the normalized specific force-velocity curve (F-V curve) obtained by the model using the parameters resulting from the fitting (see Table 2) against the experimental curve reported in Coirault et al. (2003). The stress $\sigma$ is equal to the total stress $\sigma_{\text {tot }}, \sigma_{\max }$ is the maximum stress in isometric condition, the velocity $V$ is equal to $d \lambda / d t$ and $V_{\max }$ is the maximum contraction velocity corresponding to unloaded concentric conditions. 
Table 4

Simulations results of maximum shortening velocity $\left(V_{\max }\right)$ in isotonic concentric contraction for single fiber and DIA muscle, where $V_{\max }=(d \lambda / d t)_{\min }$.

\begin{tabular}{llll}
\hline & $\begin{array}{l}V_{\max } \\
\left(s^{-1}\right)\end{array}$ & $\left.\begin{array}{l}V_{\max }(\%) \\
m d x\end{array}\right)$ & $\begin{array}{l}\text { Experiments }\left(s^{-1}\right) \\
\text { mean } \pm S D\end{array}$ \\
\hline fiber C57Bl & -8.62 & -30.50 & $-6.59 \pm 2.71$ (Pellegrino et al., 2003) \\
fiber mdx & -5.99 & & - \\
Muscle C57Bl & -8.08 & -30.72 & $-8.28 \pm 1.09$ (Coirault et al., 1999; 2003) \\
Muscle mdx & -5.59 & & $-5.13 \pm 0.99$ (Coirault et al., 1999; 2003) \\
\hline
\end{tabular}

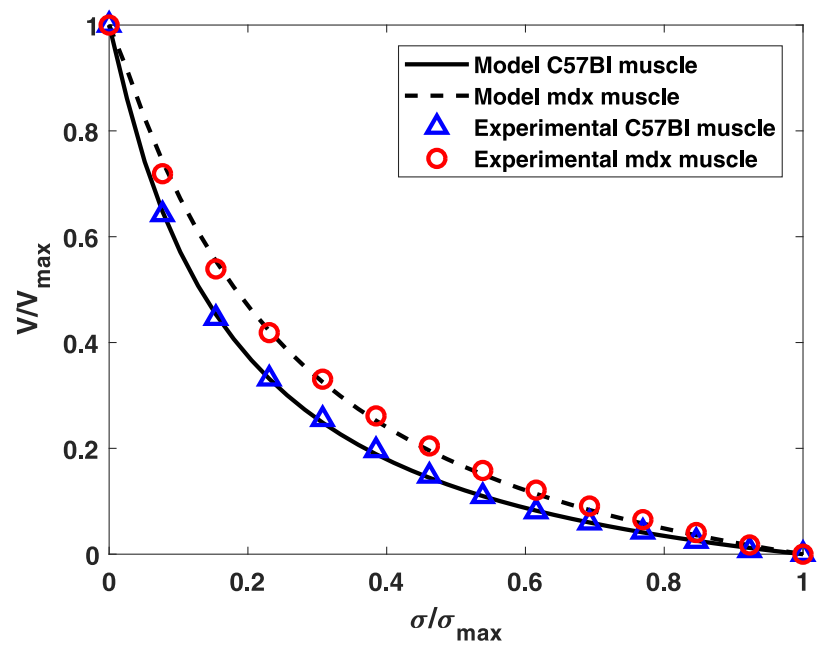

Fig. 5. Normalized specific force-velocity for C57Bl and mdx mouse diaphragm (Coirault et al., 2003).

\section{Verification of the model}

The implemented mathematical model is able to simulate isometric, concentric and eccentric uniaxial contractions. The simulation outcomes resulting from the isometric, concentric and eccentric conditions were used to verify the predictive capabilities of the model.

\subsection{Isometric and concentric contraction}

During the isometric conditions the muscle length remains constant, while the active contractile element stretch $\left(\lambda_{a}^{w}\right.$ and $\lambda_{a}^{p}$ ) decreases and the passive contractile element stretch $\left(\lambda_{e}^{w}\right.$ and $\left.\lambda_{e}^{p}\right)$ increases, due to the elastic response of the cross-bridges, titin and ECM. In addition, no passive contributions appear in the isometric contraction and so the total stress is completely an active stress. Instead, the concentric contraction happens when the muscle lifts a dead load less than the maximum force the muscle can exert. At first, the total stretch remains constant and the active stress increases progressively until equilibrating the external load. When the muscle contraction force becomes larger than the external load, the muscle can shortens. This shortening is due to the active stretch, $\lambda_{a}^{w}$ and $\lambda_{a}^{p}$.

An interesting result is that the F-V curve predicted by the model for healthy and dystrophic fiber is in the range of the experimental F-V curve for fast rat fibers (Type 2B) reported by Bottinelli et al. (1991) and fast mouse fiber (Type 2X and $2 \mathrm{~A}$ ) reported by Edman (2005), indicating the potential predictive capabilities of the model to simulate the fiber F-V curve (see Fig. 6). Bottinelli et al. (1991) performed the experiments on single fibers from EDL, SOL and PL muscles of 3-month-old male Wistar rats, while (Edman, 2005) dissected single fibers from the flexor digitorum brevis muscle (FDB), where 2A and 2X fiber type were predominant. Since Pellegrino et al. (2003) reported no differences for isometric stress and maximum shortening velocity of corresponding fiber types in rat vs mouse and DIA is mainly composed by fast fibers (Caiozzo et al., 2003; Guido et al., 2010), we have compared the normalized F-V curve of healthy fiber and muscle, obtained for mouse DIA, with the experiments data for rat.

\subsection{Eccentric contraction}

If the muscle after an isometric contraction is stretched with a constant velocity, an external force greater than the maximum isometric force of the muscle must be applied in order to lengthen the muscle. The larger the stretch velocity, the larger the stress applied to the muscle. In this case, the simulation includes two steps. First, the muscle is fully activated due 


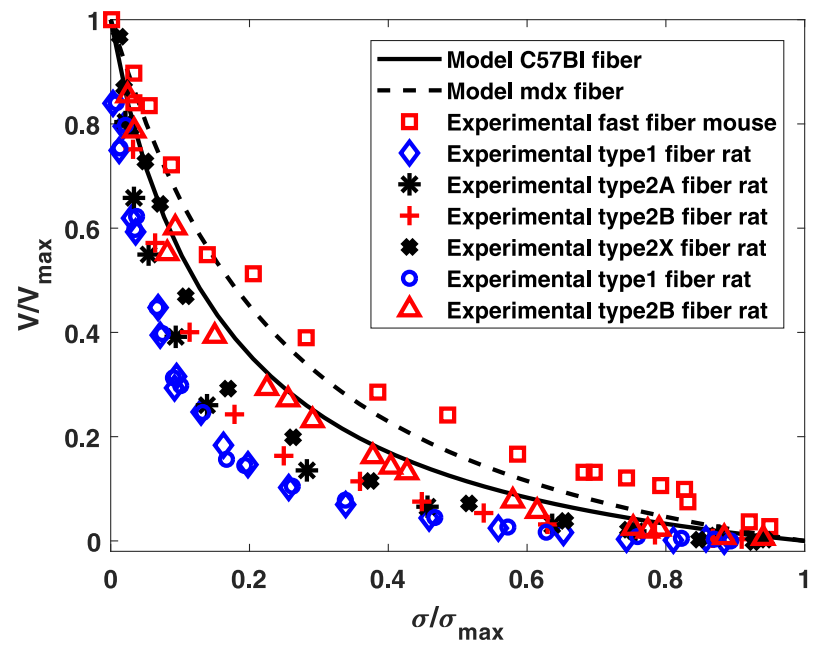

Fig. 6. Normalized F-V curve for model healthy and dystrophic fiber compared with the experimental data for slow and fast fiber (Bottinelli et al., 1991; Edman, 2005).

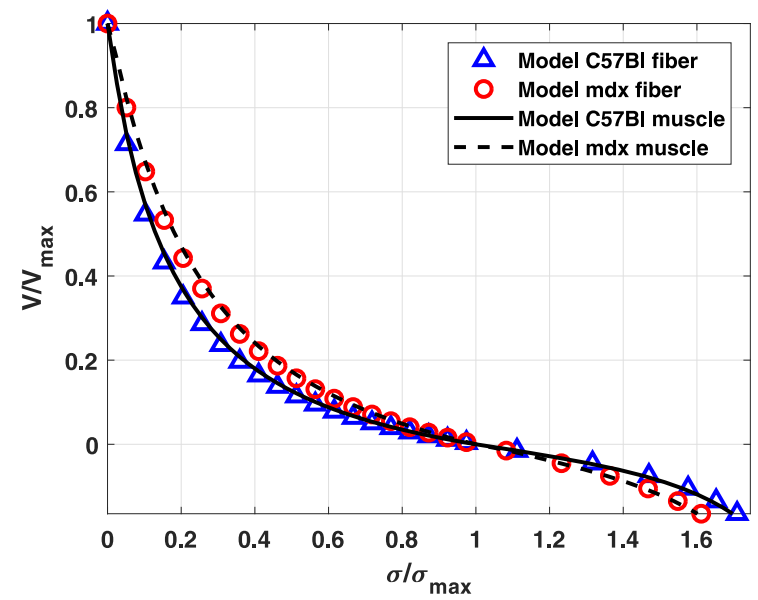

(a) Model F-V

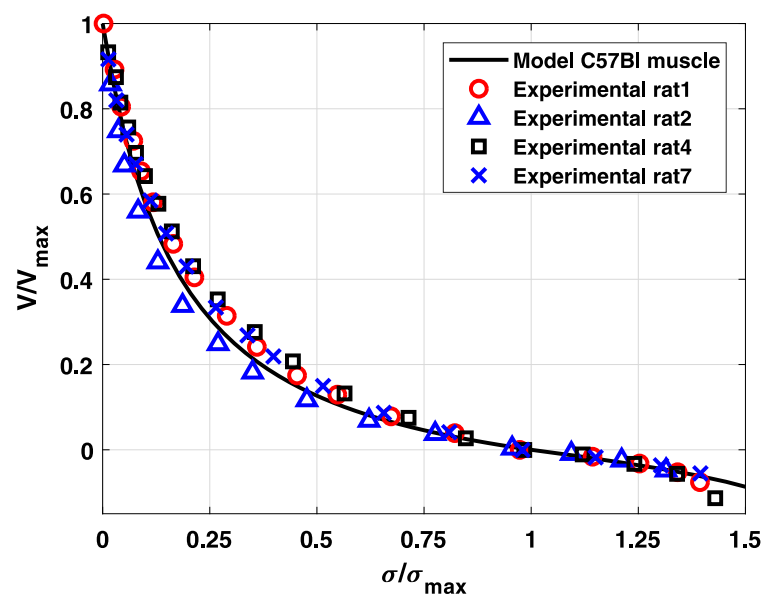

(b) Healthy muscle F-V

Fig. 7. (a) Normalized F-V curve predicted by the model for fiber and muscle in healthy and dystrophic condition. (b) Normalized F-V curve for healthy muscle compared with experimental data (Till et al., 2008). In eccentric condition $\left(V / V_{\max }<0\right)$, the muscle is stretched up to a fixed stretch of 1.04 (value in the range of experiments performed by Till et al., 2008).

to an isometric contraction and then the muscle is stretched up to a fixed stretch equal to 1.04 with a constant deformation velocity $(d \varepsilon / d t)$. During the isometric phase, the total stretch remains constant and the total stress increases progressively. Subsequently, the muscle is stretched and so $\lambda, \lambda_{a}^{w}, \lambda_{a}^{p}, \lambda_{e}^{w}$ and $\lambda_{e}^{p}$ increase. Moreover, there is an increment in passive stress due to muscle stretching.

Fig. 7a shows the normalized F-V curve predicted by the model in concentric and eccentric contraction for the fiber and the muscle. Fig. 7b shows the concentric-eccentric F-V curve of healthy muscle compared against the experimental F-V curves reported by Till et al. (2008). Experimental data corresponds to gastrocnemius medialis muscle (2 type fast fibers) of adult male Wistar rats by Till et al. (2008) during concentric isotonic and eccentric isovelocity contractions. The model predicts F-V curves within the experimental range for concentric contraction, but under predicts the eccentric force at larger stretching velocities.

\subsection{Force-length relationship}

The force-length relationship in the model is the result of changes in overlap length between the actin and myosin filaments in the sarcomere. An important peculiarity of the model is that the force-length relationship is not explicitly defined in the expression of the active stress, as usually proposed in other models (Blemker et al., 2005; Böl and Reese, 


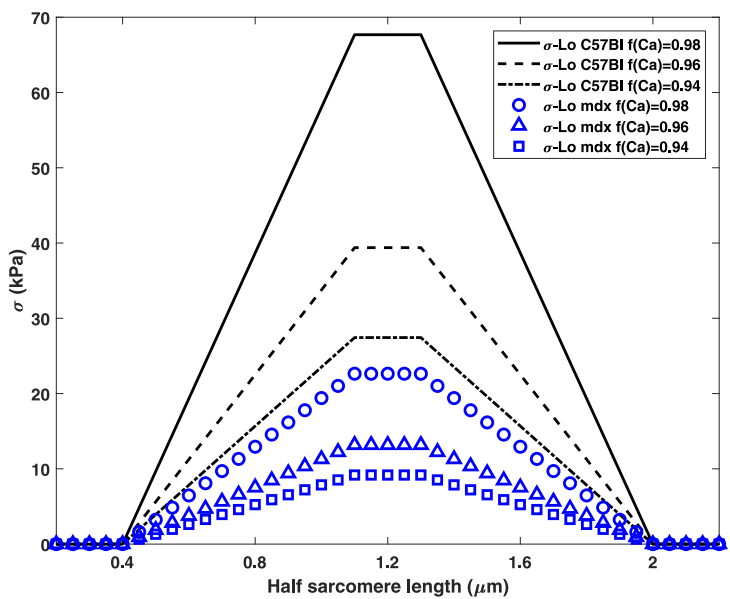

(a) $\sigma-\lambda$ curve

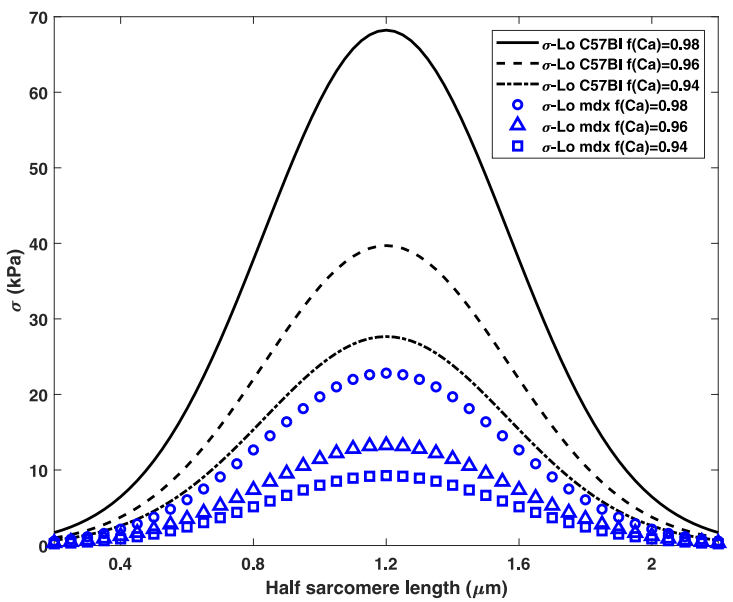

(b) $\sigma-\lambda$ curve

Fig. 8. Stress-stretch curve for $\mathrm{C} 57 \mathrm{Bl}$ and $\mathrm{mdx}$ diaphragm when calcium concentration changes. (a) Linear function. (b) Parabolic function.

Table 5

Simulations results of maximum shortening strain $\left(\varepsilon_{\min }\right)$ in isotonic concentric contraction for single fiber and DIA muscle, where $\varepsilon_{\min }=\ln \left(\lambda_{\min }\right)$.

\begin{tabular}{llll}
\hline & $\begin{array}{l}\lambda_{\min } \\
(\mathrm{mm} / \mathrm{mm})\end{array}$ & $\begin{array}{l}\varepsilon_{\min } \\
(\mathrm{mm} / \mathrm{mm})\end{array}$ & $\begin{array}{l}\varepsilon_{\min }(\%) \\
\mathrm{mdx} \text { vs C57Bl }\end{array}$ \\
\hline Fiber C57Bl & 0.6100 & -0.4943 & -20.85 \\
Fiber mdx & 0.6762 & -0.3913 & \\
Muscle C57Bl & 0.8193 & -0.1993 & -30.27 \\
Muscle mdx & 0.8703 & -0.1386 & \\
\hline
\end{tabular}

2008; Heidlauf and Röhrle, 2014; Hernández-Gascón et al., 2013). Instead, it is defined in the cross-bridge kinetics. In this way, the model takes into account that the zone in the half sarcomere overlap (Ov) in which force-bearing cross-bridges between thick and thin filaments may be form varies with sarcomere length (SL). So, the active stress is generated as a result of interaction between thick and thin filaments through cross-bridge attachment to the actin binding site on the thin filament. Fig. 8 shows the force-length curve for healthy and dystrophic model for different values of the troponin $\mathrm{C}$ subunit $(\mathrm{TnC}) \mathrm{Ca}^{2+}$ binding isotherm, $f(\mathrm{Ca})$. For a single low-affinity $\mathrm{Ca}^{2+}$ binding site, $f(\mathrm{Ca})$ is equal to the ratio (Campbell et al., 2000),

$$
f(C a)=\frac{C a(t)}{C a(t)+C a_{50}}
$$

where $\mathrm{Ca}(t)$ is the calcium bound to $\mathrm{TnC}$ of the regulatory unit (RU) troponin complex and $\mathrm{Ca}_{50}$ is the calcium concentration of half saturation of the TnC calcium binding site. Typical values of $\mathrm{Ca}(t)$ found in the cell are between $10^{-8}$ to $10^{-4} \mathrm{ML}^{-1}$, while $\mathrm{Ca}_{50}$ can be assumed equal to $1.78 \times 10^{-6} M L^{-1}$ (Campbell et al., 2000).

Currently, the force-length relationship implemented in the model is a piecewise linear function taken from Campbell et al. (2000) (see Campbell et al. (2000) (see Appendix A) and shown in Fig. 8a. The piecewise function is based on precise filaments length of $1.2 \mu \mathrm{m}$ for the thin filament and of $1.6 \mu \mathrm{m}$ for the thick filament. However, a smooth function, such as the parabolic function shown in Fig. 8b, can be used. Furthermore, it is possible include a non-symmetric forcelength curve where the stress generated drops more sharply on the ascending limb, especially after reaching thick filament length (Lieber and Ward, 2011). In all simulations the function $f(\mathrm{Ca})$ is imposed equal to 0.98 , corresponding to $10^{-4} \mathrm{ML}^{-1}$, simulating the condition in which fibers are immersed in a high $\left[\mathrm{Ca}^{2+}\right]$ concentration solution (Bates et al., 2013; Joanne et al., 2012).

\section{Discussion and conclusions}

Results from the mathematical model show that the model is able to reproduce the experimental data of maximum isometric stress and maximum contraction velocity for the healthy and dystrophic DIA, accounting for fibrosis or fat infiltration reported in literature. For the parameters in Table 2, the maximum isometric force was found to be $67.70 \mathrm{kPa}$ and $22.65 \mathrm{kPa}$ 
for the healthy and dystrophic muscles respectively, both within the range of $67.69 \pm 18.69$ and $22.56 \pm 1.70 \mathrm{kPa}$ reported in literature (Bates et al., 2013) for healthy and dystrophic muscles, respectively.

The maximum contraction velocity predicted by the model for the whole muscle was 8.08 and $5.59 \mathrm{~mm} / \mathrm{mm} / \mathrm{s}$ for healthy and dystrophic muscle respectively, both in the range of $8.28 \pm 1.09$ and $5.13 \pm 0.99 \mathrm{~mm} / \mathrm{mm} / \mathrm{s}$ reported in literature (Coirault et al., 1999; 2003) for healthy and dystrophic muscles, respectively. Furthermore, at fiber level the model provides for dystrophic fibers a 58\% reduction in maximum isometric stress, and a $21 \%$ reduction in maximum shortening strain with respect to the healthy fibers. While, the model provides for dystrophic fibers a reduction in maximum contraction velocity of $31 \%$ with respect to healthy fibers in a similar way as occurring at muscular level. At muscle scale, the model provides for the dystrophic DIA a $67 \%$ reduction in maximum isometric stress, a 31\% reduction in maximum contraction velocity, and a 30\% reduction in maximum shortening strain with respect to the healthy muscle (see Table 5). These results are in good agreement with the values reported in Bates et al. (2013) and other studies by Muller et al. (2001) and Coirault et al. (1999) confirm the soundness of the proposed formulation.

Another important aspect of this work is that the model is able to predict the force-velocity response of the muscle to eccentric loading without explicitly imposing its functional form in the formulation, as proposed by other works in the literature (Böl and Reese, 2008; Grasa et al., 2011; Heidlauf and Röhrle, 2014). In fact, the healthy muscle model is able to reproduce the experimental F-V curve of rat gastrocnemius medialis in eccentric condition reported by Till et al. (2008). In addition, the model is able to reproduce, for the healthy fiber, a concentric F-V curve in the range of the experimental data for fast fiber (Bottinelli et al., 1991; Edman, 2005). This result demonstrates the capabilities of the model to reliably represent conditions and/or scenarios that have not been considering in the parameter identification process.

Regarding the formulation, the proposed model includes a more biophysical representation of the muscle mechanics with respect to other models found in the literature. In this regard, an important characteristic of the model is that the isometric activation, the force-velocity, and the force-length relationships are not explicitly defined in the expression of the active stress, as commonly found in other models (Blemker et al., 2005; Böl and Reese, 2008; Heidlauf and Röhrle, 2014; Hernández-Gascón et al., 2013). In the present model, these relationships are intrinsically defined in the cross-bridge kinetics and the elasticity of the contractile elements i.e., the myofilaments.

However, the most notable result is that the main difference in the active behavior parameters between healthy and dystrophic models is found in the value of the stiffness related to the active element (i.e. myotubes, see parameter $\eta_{2}$ in Eq. (13)). The value of $\eta_{2}$ was found to be more than two times larger for the healthy fiber than for the dystrophic fiber indicating a significant reduction of the active stress and concentric velocity in the dystrophic fiber. This result seems to reject the hypothesis that the force in single fiber is not affected by the pathology, as reported by Bates et al. (2013) for the MDX diaphragm. This difference in $\eta_{2}$ can be associated with alterations in the fiber microstructure, specifically in the myofibril alignment within the muscle fiber, with a lower value of $\eta_{2}$ indicating larger dispersion. In a recent work, Schneidereit et al. (2018) have studied the myofibrillar disorder in single fiber of mdx EDL by means of second harmonic generation confocal microscopy. They measured the degree of local angular deviation of myofibrillar bundles from the main fiber axis (myofibrillar parallelism) finding a significant myofibril disorganization in the dystrophic case with respect to the healthy case where highly aligned bundles were found. They also found a significant correlation between the loss of myofibrillar alignment and the reduction in contractile force in mdx fiber.

Further improvement of the model will consider the myofibrils missalignment within the fiber bundle. In addition, we will include fast and slow contracting fibers in order to accurately reproduce the shortening velocity at muscle level, allowing for the muscle to be composed of different fiber types. Further, the inclusion of different types of fibers also allows the identification of potential mechanisms behind the loss of muscle functioning.

The resulting model represents a novel approach to study DMD pathogenesis by providing insights into the biophysical mechanisms regulating muscle contraction associated with the impaired muscle functionality. The formulation is amenable for implementation within a finite element software that improves its potential use for researchers to study muscle response under conditions that are not possible to explore in the laboratory. In this regard, future efforts will attempt to model the effects of absence of the mechanical link with the sarcolemma at the meso-scale, fiber-to-matrix volume ratio and ECM properties on muscle architectural properties, isometric force and shortening velocity in the dystrophic condition.

However, the current model is not exempt from limitations. First, it does not include specific aspects of the muscle micro-structure that could play a significant role on the mechanical response of the dystrophic muscle. Future investigations will study the fiber-ECM interaction by looking into changes in the micro-structure of the muscle during monoaxial loading when subjected to controlled mechanical deformation. These observations will allow to formulate a much more accurate model of the tissue, as well as to determine potential mechanisms of damage that compromise muscular functioning in DMD. Another limitation is that the passive strain energy function of the ECM, obtained by fitting the experimental uniaxial stress-strain curves, could be affected by differences in biomechanical properties resulting from the different number of fibers presented in the healthy and dystrophic DIA. So, a more accurate description should include the evaluation of the ECM mechanical behaviour alone. To overcome this limitation, future investigations will consider the mechanical characterization of the ECM by means of uniaxial tests on decellularized samples harvested from healthy and mdx DIA. Decellularization of muscle tissue through enzymatic processes and detergent washes allows to remove the fibers, maintaining the overall ECM architecture, while preserving the biomechanical properties nearly identical to those of intact muscles (Porzionato et al., 2015). Data obtained from the decellularized samples can then be used to determine the total contribution of ECM to the passive mechanical response of the muscle. 
Last but not least, extending the current three-dimensional chemo-mechanical mathematical model of the dystrophic muscle to consider the immune response in DMD muscle could result of interest for studying the progression of the pathology. In order to develop the immune-mechanical model, it could be useful to build a detailed dataset of the disease course and immune response in DMD. The disease course could be determined by tracking the evolution of passive stress-strain mechanical properties of the healthy and mdx DIA, while the evolution of the immune response could be evaluate by measuring the concentration of immune cells, macrophages, as well as the density of normal, damage and regenerating fibers at different ages. The immuno-mechanical model could represents a novel approach to study DMD pathogenesis as well as to identify and evaluate potential therapeutic targets to reduce the muscle atrophy in disease condition. Furthermore, the immuno-mechanical model could focus on identifying new dynamic changes taking place in specific pathways of immune system and its contribution to inflammation, fibrosis, stiffness, weakness and normal versus pathological muscle repair.

\section{Declaration of Competing Interest}

None.

\section{Appendix A}

Here for completeness, all the cross-bridges dynamics equations, used to implement the model of healthy and dystrophic skeletal muscle, are reported. The equations are similar of those reported by Campbell et al. (2000, 2001), but they are adapted in order to make them compatible with our developed model.

\begin{tabular}{|c|c|}
\hline \multicolumn{2}{|c|}{ Variables } \\
\hline $\mathrm{Ca}(t)$ & Time course of free activator calcium available to myofilaments \\
\hline$D(t)$ & Time course of $X B$ in the detached state \\
\hline$A_{1}(t)$ & Time course of $\mathrm{XB}$ in the attached, pre-power stroke $A_{1}$ state \\
\hline$A_{2}(t)$ & Time course of XB in the attached, post-power stroke $A_{2}$ state \\
\hline \multicolumn{2}{|c|}{ Structural Parameters } \\
\hline $\mathrm{XB}$ & Myosin cross-bridge \\
\hline $\mathrm{SL}$ & Half sarcomere length \\
\hline$L_{A}$ & Length of thin filament \\
\hline$L_{M}$ & Length of thick filament \\
\hline$B$ & Bare zone on thick filament \\
\hline$O v$ & Filament overlap zone allowing XB cycling \\
\hline$R_{T}$ & Total number of sites for $\mathrm{XB}$ attachment on half thick filament \\
\hline$\overline{R_{Z}}$ & Number of sites available for $\mathrm{XB}$ attachment within $\mathrm{Ov}$ \\
\hline$x_{0}$ & Distortion of $\mathrm{XB}$ induced by power stroke \\
\hline$L_{0}$ & Chosen half-sarcomere length \\
\hline$S L_{0}$ & Optimal half-sarcomere length \\
\hline$\overline{A_{s}}$ & Cross section area of a representative sarcomere \\
\hline \multicolumn{2}{|c|}{ Kinetic Parameters } \\
\hline$C a_{50}$ & Calcium concentration of half saturation of RU \\
\hline$k_{\text {on }}^{0}$ & Rate coefficient regulating switching on of RU when no calcium is bound \\
\hline$k_{o n}^{C a}$ & Rate coefficient regulating switching on of RU when calcium is bound \\
\hline$k_{o f f}^{0}$ & Rate coefficient regulating switching off of RU when no calcium is bound \\
\hline$k_{o f f}^{C a}$ & Rate coefficient regulating switching off of RU when calcium is bound \\
\hline$f$ & Rate coefficient regulating forward XB attachment step \\
\hline$\overline{f^{\prime}}$ & Rate coefficient regulating backward XB attachment step \\
\hline $\bar{h}$ & Rate coefficient regulating forward XB power stroke \\
\hline$\overline{h^{\prime}}$ & Rate coefficient regulating backward XB power stroke \\
\hline$g$ & Rate coefficient regulating forward XB detachment step \\
\hline$u$ & Parameter grading strength of RU-RU nearest neighbor interaction \\
\hline$w$ & Parameter grading strength of XB-RU nearest neighbor interaction \\
\hline$v$ & Parameter grading strength of $\mathrm{XB}-\mathrm{XB}$ nearest neighbor interaction \\
\hline
\end{tabular}


Definition of myofilament kinetics parameters.

$$
S L=\lambda \cdot L_{0}, \lambda=\exp \left(\varepsilon_{\log }\right), \varepsilon_{\log }=\ln (\lambda) .
$$

Initialization of Variables.

$$
\begin{aligned}
& D(0)=0, A_{1}(0)=0, A_{2}(0)=0, \\
& x_{1}^{0}=\ln \left(\lambda_{0}\right)-s_{r}^{w}, x_{2}^{0}=\ln \left(\lambda_{0}\right)-s_{r}^{p}, \lambda_{0}=1 .
\end{aligned}
$$

Time-dependent system variables: $x_{1}(t)$ and $x_{2}(t)$ represent the average elastic logarithmic deformations of XB in the $A_{1}$ and $A_{2}$ states; while, $s_{1}(t)$ and $s_{2}(t)$ represent the average non-elastic logarithmic deformations of $\mathrm{XB}$ in the $A_{1}$ and $A_{2}$ states.

$$
x_{1}=\ln (\lambda)-s_{1}, x_{2}=\ln (\lambda)-s_{2} .
$$

Activator Calcium Dependence.

$$
\begin{aligned}
& k_{o n}^{r e f}=k_{o n}^{0}+\left(k_{o n}^{C a}-k_{o n}^{0}\right) \cdot \frac{C a(t)}{C a(t)+C a_{50}}, \\
& k_{o f f}^{r e f}=k_{o f f}^{0}+\left(k_{o f f}^{C a}-k_{o f f}^{0}\right) \cdot \frac{C a(t)}{C a(t)+C a_{50}} .
\end{aligned}
$$

Sarcomere Length Dependence: Filament Overlap (Linear function).

$$
\begin{aligned}
& O v(\lambda)= \begin{cases}\frac{1}{2} \cdot\left[L_{M}+2 \cdot S L\right]-L_{A} & \text { if } 2 \cdot S L<2 \cdot L_{A}-B, \\
\frac{1}{2} \cdot\left[L_{M}-B\right] & \text { if } 2 \cdot L_{A}-B \leq 2 \cdot S L<2 \cdot L_{A}+B, \\
\frac{1}{2} \cdot\left[L_{M}-2 \cdot S L\right]+L_{A} & \text { if } 2 \cdot S L \geq 2 \cdot L_{A}+B,\end{cases} \\
& R_{Z}(t)=\frac{O v}{\frac{1}{2} \cdot\left(L_{M}-B\right)} \cdot R_{T}, R_{o f f}(t)=R_{Z}(t)-D(t)-A_{1}(t)-A_{2}(t) .
\end{aligned}
$$

Sarcomere Length Dependence: Coefficient of Length-dependent Attachment.

$$
f_{0}^{r e f}=f_{0}, f_{0}^{\prime r e f}=f_{0}^{\prime} .
$$

State Variable-Dependent Coefficients: Neighbor Interactions (RU-RU, XB-RU and XB-XB). The values of $u, w$ and $v$ are imposed equal to 1 .

$$
\begin{aligned}
k_{\text {on }}= & k_{o n}^{\text {ref }} \cdot\left\{1+\frac{D(t)}{R_{Z}(t)} \cdot[u-1]\right. \\
+ & \frac{A_{1}(t)}{R_{Z}(t)} \cdot\left[u \cdot \exp \left(L_{0} \cdot(w-1) \cdot \frac{\varepsilon_{\log }-s_{1}}{x_{0}}\right)-1\right] \\
+ & \left.\frac{A_{2}(t)}{R_{Z}(t)} \cdot\left[u \cdot \exp \left(L_{0} \cdot(w-1) \cdot \frac{\varepsilon_{\log }-s_{2}}{x_{0}}\right)-1\right]\right\}^{2}, \\
k_{\text {off }}= & k_{\text {off }}^{\text {ref }} \cdot\left\{1+\frac{D(t)}{R_{Z}(t)} \cdot\left[\frac{1}{u}-1\right]\right. \\
& +\frac{A_{1}(t)}{R_{Z}(t)} \cdot\left[\frac{1}{u} \cdot \exp \left(-L_{0} \cdot(w-1) \cdot \frac{\varepsilon_{\log }-s_{1}}{x_{0}}\right)-1\right] \\
& \left.+\frac{A_{2}(t)}{R_{Z}(t)} \cdot\left[\frac{1}{u} \cdot \exp \left(-L_{0} \cdot(w-1) \cdot \frac{\varepsilon_{\log }-s_{2}}{x_{0}}\right)-1\right]\right\}^{2}, \\
f_{0}^{r e f} & \cdot\left\{1+\frac{A_{1}(t)}{R_{Z}(t)} \cdot\left[\exp \left(L_{0} \cdot(v-1) \cdot \frac{\varepsilon_{\log }-s_{1}}{x_{0}}\right)-1\right]\right. \\
& \left.+\frac{A_{2}(t)}{R_{Z}(t)} \cdot\left[\exp \left(L_{0} \cdot(v-1) \cdot \frac{\varepsilon_{\log }-s_{2}}{x_{0}}\right)-1\right]\right\}^{2}, \\
f^{\prime}= & f_{0}^{\prime r e f} \cdot\left\{1+\frac{A_{1}(t)}{R_{Z}(t)} \cdot\left[\exp \left(-L_{0} \cdot(v-1) \cdot \frac{\varepsilon_{\log }-s_{1}}{x_{0}}\right)-1\right]\right. \\
& \left.+\frac{A_{2}(t)}{R_{Z}(t)} \cdot\left[\exp \left(-L_{0} \cdot(v-1) \cdot \frac{\varepsilon_{\log }-s_{2}}{x_{0}}\right)-1\right]\right\}^{2} .
\end{aligned}
$$

State Variable-Dependent Coefficients: Distortion dependent coefficients.

$$
h=h_{\text {iso }}, h^{\prime}=h_{\text {iso }}^{\prime}, g=g_{0} .
$$




\section{References}

Attal, P., Lambert, F., Marchand-adam, S., Bobin, S., Pourny, J.C., Chemla, D., Lecarpentier, Y., Coirault, C., 2000. Severe mechanical dysfunction in pharyngeal muscle from adult MDX mice. Am. J. Respir. Crit. Care Med. 162 (1), 278-281.

Barbin, I.C.C., Pereira, J.A., Bersan Rovere, M., de Oliveira Moreira, D., Marques, M.J., Santo Neto, H., 2016. Diaphragm degeneration and cardiac structure in MDX mouse: potential clinical implications for duchenne muscular dystrophy. J. Anat. 228 (5), 784-791.

Bates, G., Sigurdardottir, S., Kachmar, L., Zitouni, N.B., Benedetti, A., Petrof, B.J., Rassier, D., Lauzon, A.M., 2013. Molecular, cellular, and muscle strip mechanics of the MDX mouse diaphragm. Am. J. Physiol. Cell Physiol. 304, C873-C880.

Blemker, S.S., Pinsky, P.M., Delp, S.L., 2005. A 3d model of muscle reveals the causes of nonuniform strains in the biceps brachii. J. Biomech. 38 (4), 657-665.

Böl, M., Reese, S., 2008. Micromechanical modelling of skeletal muscles based on the finite element method. Comput. Methods Biomech. Biomed. Eng. 11 (5), 489-504

Bottinelli, R., Schiaffino, S., Reggiani, C., 1991. Force-velocity relations and myosin heavy chain isoform compositions of skinned fibres from rat skeletal muscle.. J. Physiol. (Lond.) 437, 655-672.

Caiozzo, V.J., Baker, M.J., Huang, K., Chou, H., Wu, Y.Z., Baldwin, K.M., 2003. Single-fiber myosin heavy chain polymorphism: how many patterns and what proportions? Am. J. Physiol. Regulat. Integrat. Comparat. Physiol. 285 (3), R570.

Campbell, K.B., Razumova, M.V., Kirkpatrick, R.D., Slinker, B.K., 2000. Variably Activated Sarcomere Model for Muscle Contraction Studies and Applications. Washington State University. Thesis.

Campbell, K.B., Razumova, M.V., Kirkpatrick, R.D., Slinker, B.K., 2001. Nonlinear myofilament regulatory processes affect frequency-dependent muscle fiber stiffness.. Biophys. J. 81 (4), 2278-2296.

Coirault, C., Lambert, F., Marchand-Adam, S., Attal, P., Chemla, D., Lecarpentier, Y., 1999. Myosin molecular motor dysfunction in dystrophic mouse diaphragm. Am. J. Physiol. Cell Physiol. 277 (46), C1170-C1176.

Coirault, C., Pignol, B., Cooper, R.N., Butler-Browne, G., Chabrier, P.E., Lecarpentier, Y., 2003. Severe muscle dysfunction precedes collagen tissue proliferation in mdx mouse diaphragm. J. Appl. Physiol. 94, 1744-1750.

Durbeej, M., Campbell, K.P., 2002. Muscular dystrophies involving the dystrophin - glycoprotein complex: an overview of current mouse models. Current Opin. Genet. Devel. 12, 349-361. doi:10.1016/S0959-437X(02)00309-X.

Edman, K.A.P., 2005. Contractile properties of mouse single muscle fibers, a comparison with amphibian muscle fibers. J. Exp. Biol. 208 (10), 1905-1913.

Emery, A.E., 2002. The muscular dystrophies. The Lancet 359 (9307), 687-695.

Farini, A., Meregalli, M., Belicchi, M., Battistelli, M., Parolini, D., D’antona, G., Gavina, M., Ottoboni, L., Constantin, G., Bottinelli, R., 2007. T and b lymphocyte depletion has a marked effect on the fibrosis of dystrophic skeletal muscles in the SCID/MDX mouse. J. Pathol. 213, 229-238.

Farini, A., Sitzia, C., Cassani, B., Cassinelli, L., Rigoni, R., Colleoni, F., Fusco, N., Gatti, S., Bella, P., Villa, C., 2016. Therapeutic potential of immunoproteasome inhibition in duchenne muscular dystrophy. Mol. Ther. 24 (11), 1898-1912.

Friedrich, O., Both, M., Weber, C., Schürmann, S., Teichmann, M.D.H., Von Wegner, F., Fink, R.H.A., Vogel, M., Chamberlain, J.S., Garbe, C., 2010. Microarchitecture is severely compromised but motor protein function is preserved in dystrophic MDX skeletal muscle. Biophys. J. 98 (4), 606-616.

Granzier, H.L., Irving, T.C., 1995. Passive tension in cardiac muscle: contribution of collagen, titin, microtubules, and intermediate filaments. Biophys. J. 68 (3), 1027-1044.

Grasa, J., Ramirez, A., Osta, R., Muñoz, M., Soteras, F., Calvo, B., 2011. A 3d active-passive numerical skeletal muscle model incorporating initial tissue strains. validation with experimental results on rat tibialis anterior muscle. Biomech. Model Mechanobiol. 10 (5), 779-787.

Guido, A.N., Campos, G.E.R., Neto, H.S., Marques, M.J., Minatel, E., 2010. Fiber type composition of the sternomastoid and diaphragm muscles of dystrophin-deficient MDX mice. Anatom. Record Adv. Integrat. Anat. Evolut. Biol. 293 (10), 1722-1728.

Hakim, C.H., Grange, R.W., Duan, D., 2011. The passive mechanical properties of the extensor digitorum longus muscle are compromised in 2-to 20-mo-old mdx mice. J. Appl. Physiol. 110 (6), 1656-1663.

Heidlauf, T., Röhrle, O., 2014. A multiscale chemo-electro-mechanical skeletal muscle model to analyze muscle contraction and force generation for different muscle fiber arrangements. Front Physiol. 5 (498), 1-14.

Hernández-Gascón, B., Grasa, J., Calvo, B., Rodríguez, J., 2013. A 3d electro-mechanical continuum model for simulating skeletal muscle contraction. J. Theor. Biol. 335, 108-118.

Hoffman, E.P., Brown Jr., R.H., Kunkel, L.M., 1987. Dystrophin: the protein product of the duchenne muscular dystrophy locus. Cell 51 (6), 919-928. doi:10. 1016/0092-8674(87)90579-4.

Joanne, P., Hourdé, C., Ochala, J., Caudéran, Y., Medja, F., Vignaud, A., Mouisel, E., Hadj-Said, W., Arandel, L., Garcia, L., 2012. Impaired adaptive response to mechanical overloading in dystrophic skeletal muscle. PLoS One 7 (4), e35346.

Khayyeri, H., Longo, G., Gustafsson, A., Isaksson, H., 2016. Comparison of structural anisotropic soft tissue models for simulating achilles tendon tensile behaviour. J. Mech. Behav. Biomed. Mater 61, 431-443.

Krüger, E., Kloetzel, P.M., 2012. Immunoproteasomes at the interface of innate and adaptive immune responses: two faces of one enzyme. Curr. Opin. Immunol. $24(1), 77-83$.

Kumamoto, T., Fujimoto, S., Ito, T., Horinouchi, H., Ueyama, H., Tsuda, T., 2000. Proteasome expression in the skeletal muscles of patients with muscular dystrophy. Acta Neuropathol. 100 (6), 595-602.

Lieber, R.L., Ward, S.R., 2011. Skeletal muscle design to meet functional demands. Philosoph. Trans. R. Soc. B Biol. Sci. 366 (1570), 1466-1476.

Lynch, G.S., Hinkle, R.T., Faulkner, J.A., 2000. Power output of fast and slow skeletal muscles of MDX (dystrophic) and control mice after clenbuterol treatment. Exp. Physiol. 85 (3), 295-299.

Muller, J., Vayssiere, N., Royuela, M., Leger, M., Muller, A., Bacou, F., Pons, F., Hugon, G., Mornet, D., 2001. Comparative evolution of muscular dystrophy in diaphragm, gastrocnemius and masseter muscles from old male MDX mice. J. Muscle Res. Cell. Motil. 22 (2), 133-139.

Pellegrino, M.A., Canepari, M., Rossi, R., D’antona, G., Reggiani, C., Bottinelli, R., 2003. Orthologous myosin isoforms and scaling of shortening velocity with body size in mouse, rat, rabbit and human muscles. J. Physiol. (Lond.) 546 (3), 677-689.

Petrof, B.J., Shrager, J.B., Stedman, H.H., Kelly, A.M., Sweeney, H.L., 1993. Dystrophin protects the sarcolemma from stresses developed during muscle contraction.. Proc. Natl. Acad. Sci. USA 90, 3710-3714.

Petrof, B.J., Stedman, H.H., Shrager, J.B., Eby, J., Sweeney, H., Kelly, A.M., 1993. Adaptations in myosin heavy chain expression and contractile function in dystrophic mouse diaphragm. Am. J. Physiol. Cell Physiol. 265 (3), C834-C841.

Porzionato, A., Sfriso, M.M., Pontini, A., Macchi, V., Petrelli, L., Pavan, P.G., Natali, A.N., Bassetto, F., Vindigni, V., De Caro, R., 2015. Decellularized human skeletal muscle as biologic scaffold for reconstructive surgery. Int. J. Mol. Sci. 16 (7), 14808-14831.

Ramaswamy, K.S., Palmer, M.L., van der Meulen, J.H., Renoux, A., Kostrominova, T.Y., Michele, D.E., Faulkner, J.A., 2011. Lateral transmission of force is impaired in skeletal muscles of dystrophic mice and very old rats. J. Physiol. (Lond.) 589 (5), 1195-1208. doi:10.1113/jphysiol.2010.201921.

Razumova, M.V., Bukatina, A.E., Campbell, K.B., 1999. Stiffness-distortion sarcomere model for muscle simulation. J Appl Physiol 87 (5), $1861-1876$.

Schneidereit, D., Nübler, S., Prölß, G., Reischl, B., Schürmann, S., Müller, O.J., Friedrich, O., 2018. Optical prediction of single muscle fiber force production using a combined biomechatronics and second harmonic generation imaging approach. Light Sci. Appl. 7 (1), 79.

Simo, J.C., Taylor, R.L., 1991. Quasi-incompressible finite elasticity in principal stretches. continuum basis and numerical algorithms. Comput. Methods Appl. Mech. Eng. 85 (3), 273-310.

Smith, L.R., Barton, E.R., 2014. Collagen content does not alter the passive mechanical properties of fibrotic skeletal muscle in mdx mice. Am. J. Physiol. Cell Physiol. 306, C889-C898.

Stålhand, J., Klarbring, A., Holzapfel, G.A., 2011. A mechanochemical 3d continuum model for smooth muscle contraction under finite strains. J. Theor. Biol. $268(1), 120-130$. 
Stedman, H., Sweeney, H., 1991. The mdx mouse diaphragm reproduces the degenerative changes of duchenne muscular dystrophy. Nature 352 (6335), 536-539.

Tidball, J.G., Wehling-Henricks, M., 2007. The role of free radicals in the pathophysiology of muscular dystrophy. J. Appl. Physiol. 102, $1677-1686$.

Till, O., Siebert, T., Rode, C., Blickhan, R., 2008. Characterization of isovelocity extension of activated muscle: a hill-type model for eccentric contractions and a method for parameter determination. J. Theor. Biol. 255 (2), 176-187.

Virgilio, K.M., Martin, K.S., Peirce, S.M., Blemker, S.S., 2015. Multiscale models of skeletal muscle reveal the complex effects of muscular dystrophy on tissue mechanics and damage susceptibility. Int. Focus 5, 1-10. 